\title{
Acoustic Radiation Force Impulse quantitative elastography: a new noninvasive technique for the evaluation of parotid glands. A preliminary study in controls and in patients with irradiated nasopharyngeal carcinoma.
}

\author{
Iulia Badea ${ }^{1}$, Attila Tamas-Szora ${ }^{2}$, Ioana Chiorean ${ }^{3}$, Maria Crisan $^{4}$, Elisabeta Ciuleanu ${ }^{5}$, \\ Grigore Baciut ${ }^{6}$, Mindra Badea ${ }^{1}$
}

\begin{abstract}
${ }^{1}$ Department of Preventive Dentistry, "Iuliu Hatieganu” University of Medicine and Pharmacy, ${ }^{2}$ Imaging Department, “O. Fodor" Institute of Gastroenterology and Hepatology, "Iuliu Hatieganu” University of Medicine and Pharmacy, ${ }^{3}$ Department of Mathematics, Faculty of Mathematics and Informatics, Babas-Bolyai University, ${ }^{4}$ Department of Histology, "Iuliu Hatiegenu" University of Medicine and Pharmacy, ${ }^{5}$ Department of Radiotherapy, Oncologic Institute "Prof. Dr. Ion Chiricuta", ${ }^{6}$ Departemnt of Maxillofacial Surgery, "Iuliu Hatieganu" University of Medicine and Pharmacy, Cluj Napoca, Romania
\end{abstract}

\begin{abstract}
Aim: We aim to define the values of the shear wave velocity (SWV) in the normal parotid glands (PG) and to identify the modifications of tissue stiffness in patients with irradiated nasopharyngeal carcinoma. Material and methods: Sixty normal parotid glands pertaining to 30 healthy volunteers and 40 pathological parotid glands pertaining to 20 patients who had had underwent radiation therapy for nasopharyngeal carcinoma were included in this study. The patients underwent a B mode ultrasonography examination and the volume of the parotid gland was determined. A subsequent Acoustic Radiation Forde Impulse Imaging (ARFI) examination was performed and the SWV were determined for the central and the periphery of the glandular parenchyma. Results: The mean volume of the gland in the volunteers' group $(12.02 \pm 0.6 \mathrm{ml})$ was significantly higher than in the group of patients who underwent radiation therapy $(10.3 \pm 0.74 \mathrm{ml})(\mathrm{p}<0.0001)$. The SWV values in the normal group were lower compared to the patients group $(1.54 \pm 0.6 \mathrm{~m} / \mathrm{s}$ vs. $1.76 \pm 0.73 \mathrm{~m} / \mathrm{s}, \mathrm{p}<0.0001)$. Conclusion: Elastography using the ARFI technique constitutes an objective method used for the evaluation of glandular stiffness in both normal and post radiotherapy glands. The SWV values determined in patients who underwent head and neck radiotherapy are increased, indicating a greater tissue stiffness thereby implying a structural transformation of the glandular parenchyma.
\end{abstract}

Keywords: ultrasonography, elastography, ARFI, parotid gland, radiotherapy.

\section{Introduction}

Head and neck malignancies are considered to be common cancers, representing a total of $6.4 \%$ of all cancer pathologies in Europe, with the highest incidence in Central and Eastern Europe [1]. In Romania the number

Received 22.03.2015 Accepted 16.05.2015

Med Ultrason

2015, Vol. 17, No 3, 308-314

Corresponding author: Attila Tamas-Szora

19-21 Croitorilor Street,

400162 Cluj Napoca, Romania,

Phone: +40740084968 ,

E-mail: tamas-szora.attila@umfcluj.ro of head and neck malignancies has increased in the last decades, a significant number of the patients being already stage IV when admitted [2]. Next to the surgical approach, radiotherapy is considered to be an important treatment and management approach of the head and neck cancers [3].

Despite the recent advancements in the field of radiotherapy, the side effects of the irradiation of the surrounding tissues such as osteoradionecrosis, xerostomia, mucositis, and altered taste, cannot be completely avoided, thus affecting the patients quality of life $[3,4]$. Xerostomia is one of the most important oral complications of radiotherapy received for the head and neck malignancies 
and occurs as a result of the damage brought by irradiation of the salivary parenchyma $[5,6]$. The reduced flow and viscosity of the saliva is a direct result of the damage brought to the radiation sensitive glandular parenchyma and its subsequent reduced salivary gland function [6]. Studies have shown that there are definite links between the exposure of the glands to radiation and the subsequent size reduction [6] and change in the blood flow of the parotid glands [7]. The parotid gland (PG) function was found to be dose dependent $[7,8]$; the threshold beyond which the damage to the glandular parenchyma becomes prominent may lay around 30-40 Gy [4].

Ultrasonography (US) is considered to be the imaging method of choice for the initial evaluation of the PG. Due to the gland's superficial position most of its parts are accessible to high frequency linear probes $(9-18 \mathrm{MHz})$, except for its deeper medial component. The healthy parotid is characterized by a homogeneous echo structure and an echogenicity similar to that of the thyroid gland [9]. However, the sonographic picture does not reflect the secreting ability of the gland. The imaging possibilities of US in radiation-induced sialadenitis are not sufficient, and the usefulness of this method in predicting xerostomia is very limited [10].

Elastography is an ultrasonographic noninvasive, new method used for assessing the elastic properties of a tissue. Shear wave elastography (SWE) uses the acoustic radiation force impulse technique (ARFI) with successive pulses generated by the system which in turn generates shear waves (SW) in the investigated tissue A higher tissue stiffness results in faster shear wave propagation (e.g. in fibrosis). [11-14]. Some authors have previously demonstrated changes in the sonographic appearances of irradiated PGs and were in the greater part qualitative or, at best, semi quantitative [15]. To our knowledge there is no data in the literature regarding the normal values of the SWV for the PGs and about the utility of this procedure in order to make an accurate diagnosis in some diseases of the PG.

Therefore the aim of this study is to define the mean values of the SWV in the normal PG and to identify if the head and neck therapeutic irradiation has an impact on the stiffness of the salivary gland parenchyma that is reflected in SWV deviations from normal values.

\section{Material and method}

\section{Patients}

This is a prospective cross-sectional study conducted between February and April 2012. During this period 50 subjects were included and pertained to two groups. Group A consisted of 30 healthy controls (male:female =
$15: 15$, mean age of 27 years, range 19-51 years) and was used to determine the PG's normal SWV values. The inclusion criteria were represented by: no previous medication or pathologies that may have altered the function or the structure of the salivary glands, normal general health status, normal values for the saliva flow rate test, normal aspect of the glandular parenchyma during ultrasound grey scale and Doppler examination. Group B comprised 20 subjects (male:female $=10: 10$, mean age 51 years, range 28-67 years) who underwent radiation therapy for nasopharyngeal carcinoma. The inclusion criteria for this group were: normal general health status, history of radiation therapy of the head and neck region of at least 35 Gy for nasopharyngeal carcinoma in the last 6 months, reduced saliva rate flow demonstrated using a standard saliva test, no salivary gland pathologies prior to radiation. The exclusion criteria consisted of: patients aged over 70 and surgical resection of the PG.

The study was approved by the Ethics Committee of the University. All procedures were in accordance with the Committee's standards and with the Helsinki Declaration of 1975, as revised in 2013. The aim and the procedure of the study were explained to the patients and informed consent was obtained from each patient before their enrolment.

\section{Radiotherapy protocol}

All the patients in Group B underwent external radiation beam therapy using the Primus linear accelerator (Siemens, Germany) and a conventional unmodulated technique. The therapy was delivered with the help of two laterally opposed fields for the nasopharynx and upper cervical region - which extended towards the external auditory canal and included the parotids. Also an anterior cervical field with a middle shield for the lower cervical region was practiced. The total radiation dose for the nasopharynx, upper cervical area and PGs was 68-70 Gy (per 35 fractions and per 51 days). The lower cervical region received 48-50 Gy (per 25 fractions and per 37 days). Concomitant chemotherapy was also conducted. In all, each patient received 4 chemotherapy cycles, each with a time span of one week. The medication was composed of Cisplatin $100 \mathrm{mg} / \mathrm{m}^{2}$ and Epirubicin 70 $\mathrm{mg} / \mathrm{m}^{2}$, both administered intravenously on the first day of each cycle. The mean interval between the end of the radiation therapy and ARFI evaluation was $128.9 \pm 24.43$ days (range 92-162 days).

\section{Ultrasound examination}

All the examinations were performed by the same examiner (AT with 4 years experience in the field). The machine used was a Siemens Acuson S2000 equipped with a multifrequence $(8-14 \mathrm{MHz})$ linear transducer. The procedure of the examination was as follows: the subject 
was positioned in dorsal decubitus, the head was slightly extended and the examined PG faced upwards. The patients were asked to breathe normally and not to swallow during the SWV quantification.

The examination started with a $2 \mathrm{D}$ grey-scale US. Transverse and longitudinal sonograms with subsequent diameter measurements of the PG were obtained. The volume was calculated automatically by the system using the three longest diameters (measured perpendicularly) and a multiplication factor of 0.52 [16].

The SWV measurements were realized from a transversal scan in the upper third of the gland. The anatomical landmarks of the plane were: ramus of the mandible (anterior); body of the PG (posterior to the ramus); tip of the mastoid process (posterior). Transducer pressure was at a minimum level and a fixed position was maintained. The measurements were acquired by placing the predefined measurement sample (ROI) in two locations within the body of the gland: first in the peripheral subcapsular parenchyma; second in the centre of the gland (more profound but immediately adjacent to the first region). The placement of the sample avoided the main vascular branches (assessed by Colour Doppler) and the depth was no more than $1.5 \mathrm{~cm}$ from the transducer surface (automatically measured by the system) (fig 1-3). The SWV measurements were expressed in $\mathrm{m} / \mathrm{s}$. A valid measurement was considered when a numerical value was displayed by the system. For invalid measurements the system displayed "XXXX". A successful examination for any given ROI was considered when 10 valid SWV values were obtained.

\section{Saliva testing}

Stimulated saliva flow rate test was realized using the Saliva - Check Buffer (GC, Germany) test kit. The stimulated saliva rate flow was quantitatively assessed by asking the patient to chew a paraffin wax pellet for 5 minutes, in order to mechanically stimulate the salivation, and to collect the saliva in a graded standard plastic cup, continuously, for the whole duration of the testing (expressed in $\mathrm{ml} / \mathrm{min}$ ). The normal values, as provided by the test kit manufacturer, were considered to be between $1-1.6 \mathrm{ml} / \mathrm{min}$.

\section{Statistical analysis}

For each group the statistical analysis was as follows. The mean value and standard deviation (SD) of SWV were calculated separately for the ten measurements recorded in the subcapsular and central parenchyma ROI. By combining all the recorded SWV values of the homolateral peripheral and central ROI a regional- right and left - mean and SD were derived. The final means of each group were determined by all the SWV measurements recorded inside the group. The values derived from the

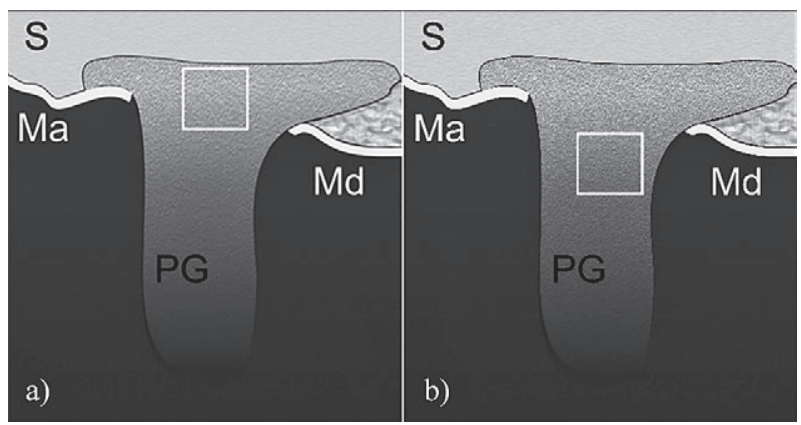

Fig 1. Drawings illustrate the transverse US scan plane and placement of the ARFI region of interest: a) ROI at subcapsular parenchyma, b) ROI at central parenchyma. White box - ARFI ROI, $\mathrm{PG}$ - parotid gland, $\mathrm{Ma}$ - mastoid, $\mathrm{Md}$ - mandible ramus, $\mathrm{S}-$ skin and subcutaneous fat.

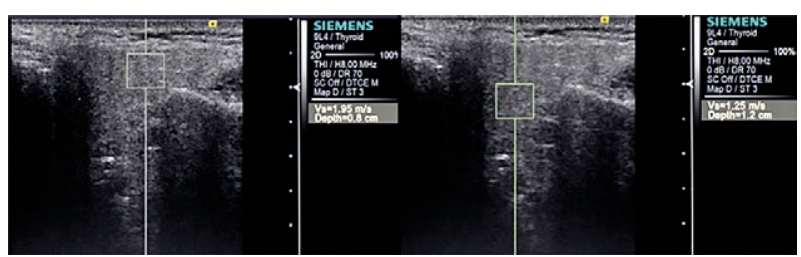

Fig 2. ARFI measurements of the left PG (transverse scan) in a 37 year old healthy control. ROI placed in the periphery and centre of the gland

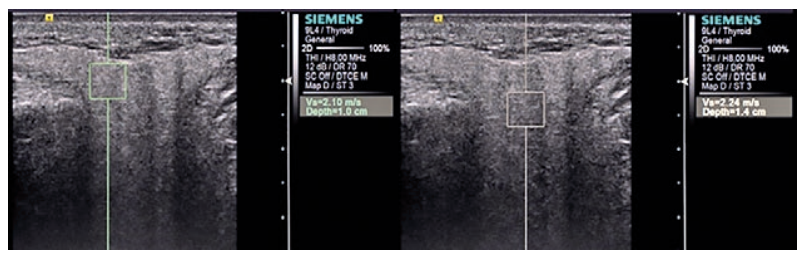

Fig 3. ARFI measurements of the left PG (transverse scan) in a 56 year old radiation therapy patient. ROI placed in the periphery and centre of the gland

stimulated saliva flow rate test were compared as means between the two groups. Microsoft Excel 2010 (Microsoft) was used for database management and MedCalc for the statistical analysis. The $\mathrm{T}$ two-tailed test with $\mathrm{n}-2$ degrees of freedom was applied to compare the mean values. The level of statistical significance was set at $\mathrm{p}<0.05$.

\section{Results}

For the stimulated saliva test a statistically significant difference was noted between the healthy controls $(8.9 \pm 1.4 \mathrm{ml} / 5 \mathrm{~min})$ and patients $(0.8 \pm 0.4 \mathrm{ml} / 5 \mathrm{~min})(\mathrm{p}$ $<0.0001$ ).

The differences between the glandular volumes of the two groups were also significant $(12.02 \pm 0.6 \mathrm{ml}$ vs $10.3 \pm 0.74 \mathrm{ml}, \mathrm{p}<0.0001)$, with a higher glandular volume for group A. 
Table I. The means and standard deviations of the SWV values determined through the ARFI technique.

\begin{tabular}{lllllll}
\hline & Left PG & & & Right PG & & \\
& Central & Peripheral & Regional & Central & Peripheral & Regional \\
\hline SWV group A $(\mathrm{m} / \mathrm{s}) \pm$ SD & $1.57 \pm 0.56$ & $1.41 \pm 0.53$ & $1.52 \pm 0.57$ & $1.51 \pm 0.48$ & $1.57 \pm 0.47$ & $1.54 \pm 0.47$ \\
SWV group B $(\mathrm{m} / \mathrm{s}) \pm \mathrm{SD}$ & $1.79 \pm 0.63$ & $1.95 \pm 0.74$ & $1.87 \pm 0.69$ & $1.47 \pm 0.41$ & $1.76 \pm 0.6$ & $1.61 \pm 0.53$ \\
\hline
\end{tabular}

PG - parotid gland

When multiplying the ten valid measurements obtained in each ROI with the number of ROIs and PGs investigated, a resultant 1200 ARFI measurements were obtained for group A and 800 for group B. The success rate for the acquisition of ARFI data was $100 \%$ (details in Table I).

When taking into account all the measurement recorded in Group A and Group B respectively, the resulted values of SWV $(1.54 \pm 0.6 \mathrm{~m} / \mathrm{s}$ vs. $1.76 \pm 0.73 \mathrm{~m} / \mathrm{s})$, presented a significant statistical difference $(\mathrm{p}<0.0001)$.No significant statistical difference was noted with respect to gender $(1.52 \pm 0.8 \mathrm{~m} / \mathrm{s}$ in female, $1.58 \pm 0.5 \mathrm{~m} / \mathrm{s}$ in male, $\mathrm{p}=0.5$ ).

\section{Discussions}

ARFI research and clinical applications for superficial structures of the head and neck has been mainly aimed at the discrimination of benignant and malignant nodules. A large proportion of the studies describe the method's diagnostic performance in thyroid cancer [1721]. Only a few papers discuss SWV's usefulness in diffuse thyroid pathologies, with moderate results $[22,23]$.

Lately, efforts have been made in the evaluation of salivary gland tumours using elastography. Bhatia et al [24] and Dumitriu et al [25] have demonstrated that although parotid malignant neoplasms are stiffer than their benign counterparts there is an important overlap between the real time elastography findings. It seems that salivary gland tumours' stiffness may vary greatly, due to their complex morphology. In other elastography studies that employed the use of SWE, results were similar, benign lesions presented with median values of $18.3 \mathrm{kPa}$ (range $0-59.4 \mathrm{kPa}$ ) and malignant lesions $13.5 \mathrm{kPa}$ (range $8-132 \mathrm{kPa})$ [26].

Iatrogenic induced PG alterations form a special category of diffuse pathology. Radiation therapy, in particular, applied for the oncological reduction of head and neck malignancies, determines irreversible glandular damage. It initially causes oedema and an acute inflammatory response, which is secondarily followed by fibrosis and gland hypotrophy [27-29]. Irreversible loss of glandular function will be encountered beginning from doses of 30-40 Gy and the saliva output after therapy is lowered with about $90 \%$ [30]. An approximate $80 \%$ of the patients that undergo radiation therapy will suffer from xerostomia, which in turn promotes infections of the oral cavity, cavities, dysphagia, speech disturbances and also pain. Overall a decrease in the quality of life is noted [27,31].

The SWE technique sheds new light on the evaluation of glandular parenchyma that may be translated into clinical applications in the field of irradiation consequences in post radiotherapy nasopharyngeal carcinoma patients, but there is very little published data concerning this problem. The elasticity of the salivary gland parenchyma probably reflects a combination of factors namely the quantity and quality of salivary glandular tissue, fatty tissue or fibrosis, which may be increased in irradiated glands [10]. The magnitude and the complexity of the affected irradiated parotid represent the motivation to identify quantifiable and reproducible methods for an early, accurate diagnosis.

The most important finding in the present study is the statistical difference in the elasticity of the salivary gland parenchyma between the post radiotherapy group (Group B) and the reference group (Group A). This can be translated as stiffer glandular parenchyma - following radiation therapy induced changes - compared to the healthy glandular structures. This finding concerning the parotid glands is in accordance with the findings of a previous study conducted by our team concerning the increase in tissue stiffness of the submandibular glands after head and neck irradiation therapy with mean SWV values of $2.13 \pm 0.52 \mathrm{~m} / \mathrm{s}$ compared to the control group with mean SWV values of $1.82 \pm 0.41 \mathrm{~m} / \mathrm{s}$ [32].

Using an identical US system as in our study, Mansour et al described the SWV values in 33 patients with parotid neoplasms. In the unaffected glands, the mean SWV was $1.75 \pm 0.64 \mathrm{~m} / \mathrm{shigher}$ than the values recorded by our study [33]. The patients included were not previously screened, as in our study, for any possible influencing conditions, and no data was provided regarding the number of measurements taken and the site of ROI placement. Another report [34], describes on a limited number of healthy controls (30 subjects), values of 1.9 $\mathrm{m} / \mathrm{s}$ for men and $1.78 \mathrm{~m} / \mathrm{s}$ for women and states no difference among values when gender or age were taken 
into account. The authors recorded five measurements in the centre and the periphery of the gland. In a similar publication context Kintworth et al state that healthy PG SWVs are $1.45 \pm 0.27 \mathrm{~m} / \mathrm{s}$, without depicting any relevant relation to gender or any differences between the left and right gland [35].

Our study has proved that in the healthy controls there is no difference between the regional means of the left and right PG, and also none between genders. However, it should be noted that we encountered a significant difference between the values measured in the periphery of the left and right glands. This finding could not be explained by our study protocol, and may be only an isolated finding as there is no proof of different histological compositions among the left and right PGs - nevertheless further attention is required.

Another point worth mentioning is the similarity between the SWVs of the superficial and more profound, central parenchyma. The comparison was made in order to establish if the stiffness of the subcapsular parenchyma is influenced in any way by the fibrous septa, which originate from the parotid fascia and descend into the glandular tissue [36]. Also we considered the comparison necessary due to the ARFI studies regarding liver stiffness, where the ROI's depth of insertion proved to influence the measured SWV [37,38].

In Group B the SWV means were quite different between the central and peripheral ROIs of the right parotid and also when compared to their left parotid counterparts. The only exception was due to the similarity of the means measured in the left PG (centre vs. periphery). The regional means of the right and left PGs were consistently different. A concise explanation for these variations is not yet at hand. Possibly confounding facts might be the increase of the gland's radiation sensitivity due to the associated chemotherapy [39], variation of radiation doses and tissue function.

Our study shows an existing characteristic decay of the saliva volume $(0.8-0.4 \mathrm{ml} / 5 \mathrm{~min})$ in patients who had underwent radiotherapy of the head and neck region. This decay is in accordance with a reduced PG volume (as measured with the US).

As our data points out, ARFI is capable of depicting radiation induced changes in the elasticity of the glandular tissue and the implied morphological alterations of the PG and thus could be of help in understanding the complex mechanisms of xerostomia. This could be further valued in the development, validation and optimization of methods that aim at improving the quality of life - such as intensity modulated radiotherapy. On the other hand, great efforts have been made to develop counteracting, radio-protective medications (aminofostine, pi- locaprine and carbacholine, coumarin, adrenergic substances) [40-42]. ARFI may, in the future, be part of the protocols aiming at evaluating response efficiency [43]. Further studies are required, with a greater number of subjects, for the verification of this hypothesis.

Other arguments that sustain ARFI's potency for the above-mentioned applications are its repeatability and cheapness.

A limitation of our study is due to the relative small sample of healthy controls and patients - which were not matched for age. Regarding the age factor, Scott et al [44] have described that age-related differences of intralobular component tissues of human PGs are consistently seen in the age group of 70-90 years - as higher percentages of adipose tissue (36.8\%) and lower percentages of glandular parenchyma (53.3\%). The age groups of $17-40$ years and 40-69 years share almost identical percentages of adipose tissue $(24.5 \%$ and $24.8 \%)$ and glandular parenchyma $(65.8 \%$ vs. $64.8 \%)$. The percentages of fibrous and vascular structures present only little variation (17-40 years $9.7 \%$, 41-69 years $10.4 \%, 70-90$ years $9.8 \%$ ). Our study group comprised of subjects less than 70 years and thus the age factor should not have had an impact on the SWV measurements between the groups. Nevertheless further studies are required for establishing the influence of age on glandular stiffness. Forthcoming studies should also address the issues of intra-/inter-observer variability, which was not obtained with our study design. Another limitation is the lack of patient SWV data before and during the different cycles of radiation therapy; therefore, we could not perform a characterisation of the SWV before and during the radiotherapy. Further studies in this direction are needed.

\section{Conclusions}

The present study is a consistent step towards filling the void in the field of salivary glands' sonographic elastography. We have achieved, in a preliminary manner, an ARFI examination protocol and the definition of SWVs for a healthy population group. The knowledge of normal values is of great importance in setting the basis for clinical acceptance of the method (in the field). Also we have statistically described the variations between the healthy and irradiated glandular parenchyma's SWVs and observed the increase in tissue stiffness related to radiation therapy changes.

\section{Acknowledgements}

This study was financially supported in part by the Iuliu Hatieganu University of Medicine and Pharmacy, 
Cluj-Napoca through the grant no. 1491 / 23 / 28.01.2014 and in part through the POSDRU no. 107/1.5/S/78702 doctoral scholarship project.

\section{Conflict of interest: none}

\section{References}

1. Hashibe M, Boffetta P, David Z, et al. Evidence for an important role of alcohol and aldehyde - metabolizing genes in cancers of the upper aerodigestive tract. Cancer Epidemiol Biomarkers Prev 2006; 15: 696-703.

2. Lung T, Tascau OC, Almasan HA, Mureşan O. Head and neck cancer, epidemiology and histological aspects - Part 1: a decade's results 1993-2002. J Craniomaxillofac Surg 2007; 35: 120-125.

3. Pesqueira AA, Goiato MC, Dos Santos DM, et al. Patients from the oral oncology center, UNESP, Aracatuba with an indication for prosthesis. Mol Clin Oncol 2013; 1: 733-736.

4. Obinata K, Nakamura M, Carrozzo M, et al. Changes in parotid gland morphology and function in patients treated with intensity-modulated radiotherapy for nasopharyngeal and oropharyngeal tumors. Oral Radiol 2014; 30: 135-141.

5. Lee TF, Chao PJ, Ting HM, et al. Using multivariate regression model with least absolute shrinkage and selection operator (LASSAO) to predict the incidence of xerostomia after intensity-modulated radiotherapy for head and neck cancer. PLoS One 2014; 9: e89700.

6. Imanimoghaddam M, Rahrooh M, Tafakhori Z, Zahedanaraki S, Homaeieshandiz F. Changes of parotid and submandibular glands caused by radiotherapy - an ultrasound evaluation. Dentomaxillofac Rad 2012; 41: 379-384.

7. Wu VW, Ying MT, Kwong DL. Evaluation of radiationinduced changes to parotid glands following conventional radiotherapy in patients with nasopharyngeal carcinoma. $\mathrm{Br}$ J Radiol 2011; 84: 843-849.

8. Yang X, Tridandapani S, Beitler JJ, Yu DS, Yoshida EJ, Curran WJ, Liu T. Ultrasound GLCM texture analysis of radiation-induced parotid-gland injury in head-and-neck cancer radiotherapy: an in vivo study of late toxicity. Med Phys 2012; 39: 5732-5739.

9. Viselner G, van der Byl G, Maira A, Merico V, Draghi F. Parotid abscess: mini-pictorial essay. J Ultrasound 2013; 16: $11-15$

10. Kaluzny J, Kopec T, Szczepanek-Parulska E, et al. Shear wave elastography: a new noninvasive tool to assess the intensity of fibrosis of irradiated salivary glands in head and neck cancer patients. Biomed Res Int 2014; 2014: 157809.

11. Schiettecatte A, Shabana W, Stadnik T, De Mey J. Inadequacy of the one-dimansional fit-model formula for ultrasound volume assessment of the thyroid gland. JBR-BTR 2006; 89: 254-257.

12. Park MK, Jo J, Kwon H, et al. Usefulness of acoustic radiation force impulse elastography in the differential diagnosis of benign and malignant pancreatic lesions. Ultrasonography $2014 ; 33: 26-33$.
13. Bamber J, Cosgrove D, Dietrich CF, et al. EFSUMB guidelines and recommendations on the clinical use of ultrasound elastography. Part 1: Basic principles and technology. U1traschall Med 2013; 34: 169-184.

14. Gennisson JL, Deffieux T, Fink M, Tanter M. Ultrasound elastography: Principles and techniques. Diagn Interv Imaging 2013; 94: 487-495.

15. Cantisani V, Lodise P, Grazhdani H, et al. Ultrasound elastography in the evaluation of thyroid pathology. Current status. Eur J Radiol 2014; 83: 420-428.

16. Kim YS, Park JG, Kim BS, Lee CH, Ryu DW. Diagnostic value of elastography using acoustic radiation force impulse imaging and strain ratio for breast tumors. J Breast Cancer 2014; 17: 76-82.

17. Zhang YF, Xu HX, He Y, et al. Virtual touch tissue quantification of acoustic radiation force impulse: a new ultrasound elastic imaging in the diagnosis of thyroid nodules. PLoS One 2012; 7: e49094.

18. Bojunga J, Dauth N, Berner C, et al. Acoustic radiation force impulse imaging for differentiation of thyroid nodules. PLoS One 2012; 7: e42735.

19. Gu J, Du L, Bai M, et al. Preliminary study on the diagnostic value of acoustic radiation force impulse technology for differentiating between benign and malignant thyroid nodules. J Ultrasound Med 2012; 31: 763-771.

20. Friedrich-Rust M, Romenski O, Meyer G, et al. Acoustic Radiation Force Impulse-Imaging for the evaluation of the thyroid gland: a limited patient feasibility study. Ultrasonics 2012; 52: 69-74.

21. Hou XJ, Sun AX, Zhou XL, et al. The application of Virtual Touch tissue quantification (VTQ) in diagnosis of thyroid lesions: A preliminary study. Eur J Radiol 2013; 82: 797-801.

22. Sporea I, Vlad M, Bota S, et al. Thyroid stiffness assessment by acoustic radiation force impulse elastography (ARFI). Ultraschall Med 2011; 32: 281-285.

23. Sporea I, Sirli R, Bota S, Vlad M, Popescu A, Zosin I. ARFI elastography for the evaluation of diffuse thyroid gland pathology: Preliminary results. World J Radiol 2012; 4: 174178.

24. Bhatia KS, Rasalkar DD, Lee YP, et al. Evaluation of real time qualitative sonoelastography of focal lesions in the parotid and submandibular glands: applications and limitations. Eur Radiol 2010; 20: 1958-1964.

25. Dumitriu D, Dudea SM, Botar-Jid C, Băciuţ G. Ultrasonographic and sonoelastographic features of pleomorphic adenomas of the salivary glands. Med Ultrason 2010; 12 : 175-183.

26. Bhatia KS, Cho CC, Tong CS, Lee YY, Yuen EH, Ahuja AT. Shear wave elastography of focal salivary gland lesions: preliminary experience in a routine head and neck US clinic. Eur Radiol 2012; 22: 957-965.

27. Epstein JB, Thariat J, Bensadoun RJ, et al. Oral complications of cancer and cancer therapy: from cancer treatment to survivorship. CA Cancer J Clin 2012; 62: 400-422.

28. Grundmann O, Mitchell GC, Limesand KH. Sensitivity of salivary glands to radiation: from animal models to therapies. J Dent Res 2009; 88: 894-903. 
29. Dörr W, Riesenbeck D. Mundhöhle. In: Dörr W, Zimmermann JS, Seegenschmiedt MH, Hrsg. Nebenwirkungen in der Radioonkologie. München: Urban \& Vogel, 2000: 130142.

30. Guchelaar HJ, Vermes A, Meerwaldt JH. Radiation-induced xerostomia: pathophysiology, clinical course and supportive treatment. Support Care Cancer 1997; 5: 281-288.

31. Jemal A, Siegel R, Ward E, Murray T, Xu J, Thun MJ. Cancer statistics, 2007. CA Cancer J Clin 2007; 57: 43-66.

32. Badea AF, Tamas Szora A, Ciuleanu E, et al. ARFI quantitative elastography of the submandibular glands. Normal measurements and the diagnosis value of the method in radiation submaxillitis. Med Ultrason 2013; 15: 173-179.

33. Mansour N, Stock KF, Chaker A, Bas M, Knopf A. Evaluation of parotid gland lesions with standard ultrasound, color duplex sonography, sonoelastography, and acoustic radiation force impulse imaging - a pilot study. Ultraschall Med 2012; 33: 283-288.

34. Guerin A, Saraux A, Cornec D, et al. Is acoustic radiation force impulse (ARFI) quantification a useful noninvasive method for assessing salivary glands? Ann Rheum Dis 2014; 73(Suppl 2):667.

35. Klintworth N, Bozzato A, Mantsopoulos K, et al. Critical evaluation of ARFI-Sonoelastogaphy of the major salivary glands. Ultraschall in Med 2013; 34 (Suppl 1): WS SL12_03.

36. Ramsaroop L, Singh B, Allopi L, Moodley J, Partab P, Satyapal KS. The surgical anatomy of the parotid fascia. Surg Radiol Anat 2006; 28: 33-37.
37. Doherty JR, Trahey GE, Nightingale KR, Palmeri ML. Acoustic radiation force elasticity imaging in diagnostic ultrasound. IEEE Trans Ultrason Ferroelectr Freq Control 2013; 60: 685-701.

38. Potthoff A, Attia D, Pischke S, et al. Influence of different frequencies and insertion depths on the diagnostic accuracy of liver elastography by acoustic radiation force impulse imaging (ARFI). Eur J Radiol 2013; 82: 1207-1212.

39. Kapanen M, Collan J, Saarilahti K, Heikkonen J, Kairemo $\mathrm{K}$, Tenhunen M. Accuracy requirements for head and neck intensity-modulated radiation therapy based on observed dose response of the major salivary glands. Radiother Oncol 2009; 93: 109-114.

40. Sauer R, Strnad V. New data on cytoprotection in radiotherapy. Strahlenther Onkol 1999; 175(Suppl 4): 1.

41. Johnson JT, Ferretti GA, Nethery WJ, et al. Oral pilocarpine for post-irradiation xerostomia in patients with head and neck cancer. N Engl J Med 1993; 329: 390-395.

42. Vacha P, Fehlauer F, Mahlmann B, et al. Randomized phase III trial of postoperative radiochemotherapy $+/$ - amifostine in head and neck cancer. Is there evidence for radioprotection? Strahlenther Onkol 2003; 179: 385-389.

43. Cotrim AP, Hyodo F, Matsumoto K, et al. Differential radiation protection of salivary glands versus tumor by tempol with accompanying tissue assessment of tempol by magnetic resonance imaging. Clin Cancer Res 2007; 13: 4928-4933.

44. Scott J. Structure and Function in Aging Human Salivary Glands. Gerodontology 1986; 5: 149-158. 